\title{
Search for Squark Production in R-parity Violating Supersymmetry at HERA
}

\section{Michael Herbst ${ }^{* \dagger}$}

University of Heidelberg, DESY-HI

E-mail: mherbstemail.desy.de

\begin{abstract}
A search for squarks in $R$-parity violating supersymmetry is performed in $e^{ \pm} p$ collisions at HERA using the $\mathrm{H} 1$ detector. The full data sample taken at a centre-of-mass energy $\sqrt{s}=319 \mathrm{GeV}$ is used for the analysis, corresponding to an integrated luminosity of $438 \mathrm{pb}^{-1}$. The resonant production of squarks via a Yukawa coupling $\lambda^{\prime}$ is considered, taking into account direct and indirect $R$-parity violating decay modes. No evidence for squark production is found in the (multi-)lepton and (multi-)jet final state topologies investigated. Mass dependent limits on $\lambda^{\prime}$ are obtained in the framework of the Minimal Supersymmetric Standard Model and in the minimal Supergravity Model. In the considered part of the parameter space, for a Yukawa-type coupling of electromagnetic strength $\lambda^{\prime}=0.3$, squarks of all flavours are excluded up to masses of $275 \mathrm{GeV}$ at $95 \%$ confidence level, with down-type squarks further excluded up to masses of $290 \mathrm{GeV}$.
\end{abstract}

XVIII International Workshop on Deep-Inelastic Scattering and Related Subjects April 19 -23, 2010

Convitto della Calza, Firenze, Italy

*Speaker.

$\dagger$ on behalf of the H1 Collaboration 


\section{Theory and Motivation}

The lepton-hadron collider HERA at DESY in Hamburg is a unique facility to study interactions in high energy collisions of electrons ${ }^{1}$ and quarks. Besides the well confirmed Standard Model (SM) physics, beyond the Standard Model (BSM) theories with new particles coupling to electron-quark pairs can also be directly tested [1]. Supersymmetric (SUSY) theories belong to the most promising theories today for BSM physics. However, the phenomenology of SUSY models strongly depends on the assumed SUSY breaking mechanism, the SUSY model parameters and on the conservation or violation of a new quantum number, denoted R-parity $R_{p}=(-1)^{3 B+L+2 S}$, where $B$ denotes the baryon number, $L$ the lepton number and $S$ the spin of a particle.

Of special interest for BSM physics at HERA are SUSY models with R-parity violation $(R / p)$, because they allow for baryon and lepton number violating process to occur. The $R_{p}^{\prime}$ Yukawa couplings especially interesting for squark production at HERA are described in the superpotential by the term $\lambda_{i j k}^{\prime} L_{i} Q_{j} \bar{D}_{k}$, where $i, j$ and $k$ are family indices. $L_{i}, Q_{j}$ and $\bar{D}_{k}$ are superfields, which contain the left-handed leptons, the left-handed quarks and the right-handed down quark. Hence single squarks could be produced resonantly via non-vanishing $R_{p}$ couplings $\lambda^{\prime}$ in the collisions of incoming $27.6 \mathrm{GeV}$ electrons and quarks from the incoming $920 \mathrm{GeV}$ proton, so that squark masses up to the electron-proton centre-of-mass energy, $\sqrt{s}=319 \mathrm{GeV}$, are kinematically accessible.

In $R_{p}$ SUSY, all supersymmetric particles are unstable. The $\tilde{d}_{R}$-type squarks can decay either into $e^{-}+u^{j}$ or $v_{e}+d^{j}$, while the $\tilde{u}_{L}$-type squarks decay into $e^{+}+d^{k}$ only. The $R_{p}$ squark decays proceed directly via the couplings $\lambda_{11 k}^{\prime}$ and $\lambda_{1 j 1}^{\prime}$ resulting in final states similar to ordinary SM processes at HERA like neutral current (NC) and charged current (CC) deep inelastic scattering (DIS). Squarks can also decay via their usual gauge couplings leading to final states analogous to final states of higher order NC and CC DIS processes. The $\tilde{u}_{L}$-type squarks can undergo a gauge decay into states involving the superpartners of SM gauge bosons, i.e. neutralinos $\chi_{i}^{0}(i=1 \ldots 4)$, charginos $\chi_{i}^{+}(i=1,2)$ and gluinos $\tilde{g}$. By contrast, $\tilde{d}_{R}$-type squarks decay to neutralinos or gluinos only and decays into charginos are suppressed.

\section{Data Analysis}

In total 8 (9) final state toplogies are distinguished for the analysis of $255 \mathrm{pb}^{-1} e^{+} p\left(183 \mathrm{pb}^{-1}\right.$ $e^{-} p$ ) collision data, based on the presence of electrons $(e)$, muons $(\mu)$, neutrinos $(v)$ and single $(q)$ or multiple jets $(M J)$ : the lepton-quark channels $e q$ and $v q$; multijet channels with exactly one isolated electron $e^{+} M J, e^{-} M J$, where the charge measurement of the electron is used; a multijet channel with missing energy due to neutrinos $v M J$; and multijet channels with multiple leptons $e e M J, e \mu M J, e v M J$ and $v \mu M J$. The event selection employed results in exclusive analysis channels. Identified high transverse momentum electrons, muons, jets and reconstructed neutrinos are required to allocate an event to a certain analysis channel. Further cuts are applied to exploit the signal event topology, where the squark decay products are predominantly emitted into the forward direction of the detector, increasing the signal to background ratio for each channel [2]. The remaining expectation is dominated by an irreducible SM background. The expected number of

\footnotetext{
${ }^{1}$ In the following the term electron refers to both electron and positron unless explicitly stated otherwise.
} 
events from SM processes, the number of selected data events and the signal selection efficiencies are given in table 1. No significant deviation from the SM expectation is observed.

\section{Interpretation}

Using a phenomenological Minimal Supersymmetric Standard Model (MSSM) and the minimal Supergravity model (mSUGRA), limits are set using a frequentist approach based on likelihood ratios. The SUSYGEN3 [3] generator is used for the calculation of branching ratios into the analysed squark decay final states as well as the simulation of signal events.

In a MSSM scenario, the masses and mixings of gauginos are defined by the three parameters: the ratio of Higgs vacuum expectation values, $\tan \beta$; the Higgs mixing parameter $\mu$; the mass scale $M_{2}$ of $S U(2)$ sparticles. Masses of squarks are free parameters and slepton masses are set to $90 \mathrm{GeV}$. Figure 1 shows the result of a scan of scenarios for $70<M_{2}<350 \mathrm{GeV}$ and $-300<\mu<300 \mathrm{GeV}$ at fixed $\tan \beta=6$ for $\lambda_{1 j 1}^{\prime}$ and $\lambda_{11 k}^{\prime}(j, k=1,2,3)$. Shown are the limits excluded at $95 \%$ CL for all investigated scenarios with a solid line and for the best exclusion achieved in all scenarios with a dashed line. Indirect limits from low energy precision observables [4] are shown as well as the limit from the previous analysis [1]. For Yukawa-type couplings $\lambda_{1 j 1}^{\prime}$ or $\lambda_{11 k}^{\prime}$ of electromagnetic strength $\sqrt{4 \pi \alpha_{\mathrm{em}}}=0.3$, up-type squarks are excluded up to masses of $275 \mathrm{GeV}$ at $95 \% \mathrm{CL}$, with down-type squarks further excluded up to masses of $290 \mathrm{GeV}$.

By contrast, all masses and mixings are determined completely by five parameters when using the mSUGRA model: the common mass of scalar sparticles $m_{0}$; the common mass of fermionic sparticles $m_{1 / 2}$; the common trilinear coupling $A_{0}$; the ratio of Higgs vacuum expectation values, $\tan \beta$; the sign of the Higgs mixing parameter $\mu$. Figure 2 shows constraints on the parameters $\left(m_{0}, m_{1 / 2}\right)$ when $R_{p}$ couplings of electromagnetic coupling strength are assumed. The hatched blue region gives the excluded domain for first and second generation squarks and the green region indicates where third generation squarks could be excluded at 95\% CL. Curves of constant squark masses and partly complementary constraints from other colliders are indicated for comparison.

The $\tan \beta$ dependence of mSUGRA exclusion limits is studied assuming a unified common mass $M=m_{0}=m_{1 / 2}$. The region below the lines in figure 3 is excluded at $95 \% \mathrm{CL}$ for couplings comparable to the electromagnetic coupling strength for each of the indicated flavours. Results for the first two generation of up-type and down-type squarks are independent of $\tan \beta$, while mixing effects with increasing $\tan \beta$ lead to a light stop (sbottom) component or higher production cross section for one component.

\section{References}

[1] A. Aktas et al. [H1 Collaboration], Eur. Phys. J. C 36 (2004) 425 [hep-ex/0403027].

[2] J. Haller, DESY-THESIS-2003-035, available at http://www-h1.desy.de/publications/theses_list.html.

[3] S. Katsanevas and P. Morawitz, Comput. Phys. Commun. 112 (1998) 227 [hep-ph/9711417];

N. Ghodbane, S. Katsanevas, P. Morawitz and E. Perez, SUSYGEN 3, [hep-ph/9909499].

[4] J. Butterworth and H. Dreiner, Nucl. Phys. B397 (1993) 3 and references therein. 


\begin{tabular}{|c|cc|cc|c|}
\hline \multicolumn{5}{|c|}{ Search for Squarks in RPV SUSY with H1 (Preliminary) } \\
\hline $\begin{array}{c}\text { Selection } \\
\text { Channel }\end{array}$ & \multicolumn{2}{|c|}{$e^{+} p\left(255 \mathrm{pb}^{-1}\right)$} & \multicolumn{2}{|c|}{$e^{-} p\left(183 \mathrm{pb}^{-1}\right)$} & $\begin{array}{c}\text { Signal } \\
\text { Data }\end{array}$ \\
\hline \hline$e q$ & 2946 & $2899 \pm 302$ & 3121 & $3215 \pm 336$ & $30-40 \%$ \\
$v q$ & - & - & 2858 & $2983 \pm 358$ & $50-60 \%$ \\
$e M J(\mathrm{RC})$ & 140 & $145.6 \pm 21.3$ & 147 & $157.7 \pm 23.8$ & $10-40 \%$ \\
$e M J(\mathrm{WC})$ & 1 & $0.6 \pm 0.4$ & 0 & $1.3 \pm 0.3$ & $5-20 \%$ \\
$v M J$ & 19 & $23.4 \pm 5.8$ & 24 & $28.9 \pm 7.2$ & $5-15 \%$ \\
$e e M J$ & 2 & $1.7 \pm 0.5$ & 0 & $1.5 \pm 0.5$ & $5-35 \%$ \\
$e \mu M J$ & 0 & $0.03 \pm 0.03$ & 0 & $0.03 \pm 0.02$ & $5-15 \%$ \\
$v e M J$ & 5 & $8.2 \pm 2.0$ & 3 & $5.6 \pm 1.2$ & $5-40 \%$ \\
$v \mu M J$ & 0 & $0.06 \pm 0.03$ & 0 & $0.04 \pm 0.02$ & $5-20 \%$ \\
\hline
\end{tabular}

Table 1: Total numbers of selected events, SM expectation and ranges of selection efficiencies for the squark decay channels considered in the analysis of $255 \mathrm{pb}^{-1} e^{+} p$ and in $183 \mathrm{pb}^{-1} e^{-} p$ collision data. The total error on the SM prediction is determined by adding the effects of all model and experimental systematic uncertainties in quadrature.
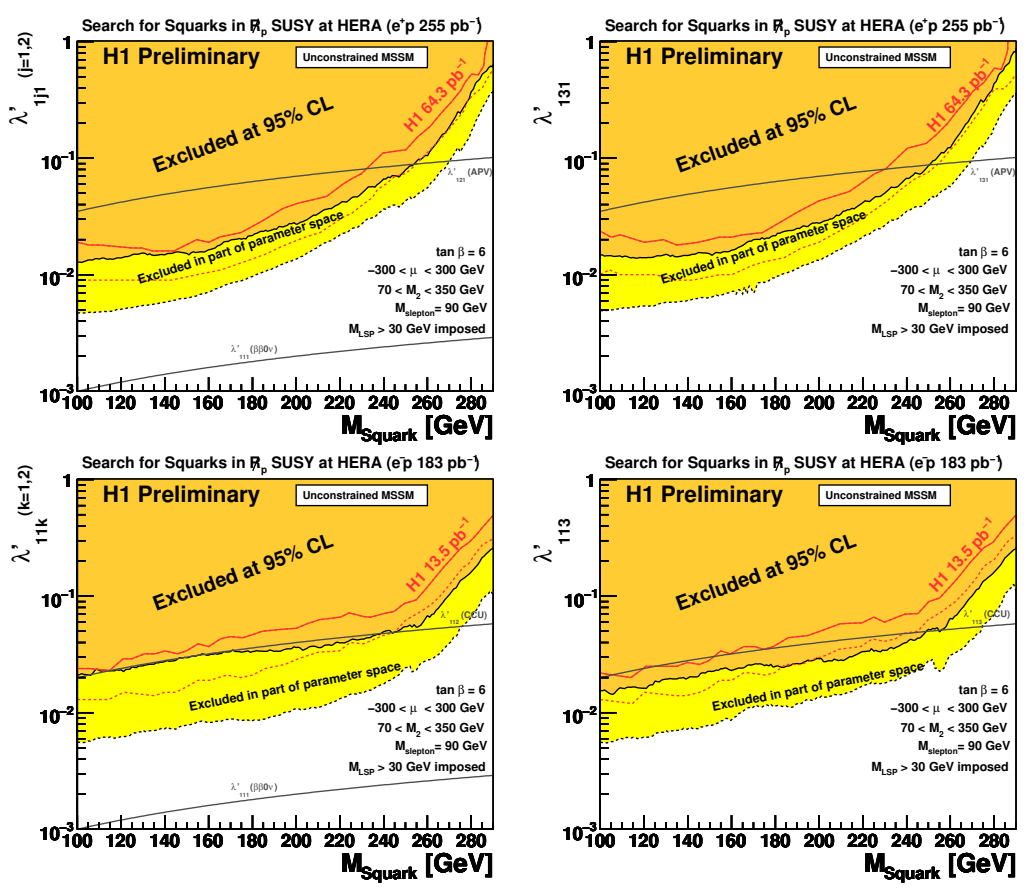

Figure 1: Exclusion limits at 95\% CL on $\lambda_{1 j 1}^{\prime}$ for (a) $j=1,2$ and (b) $j=3$ and $\lambda_{11 k}^{\prime}$ for (c) $k=1,2$ and (d) $k=3$ as a function of the squark mass from a scan of the MSSM parameter space as indicated in the figures. Values of $\lambda^{\prime}$ higher than the solid curve could be excluded in all scenarios, values in between the solid and the dashed curve could be excluded in part of the parameter space investigated. Indirect limits from neutrinoless double beta decay experiments $(\beta \beta 0 \mathrm{v})$, atomic parity violation (APV) and charged current universality (CCU) are also shown. For comparison, the corresponding limit from the HERA I analysis [1] is also indicated. 

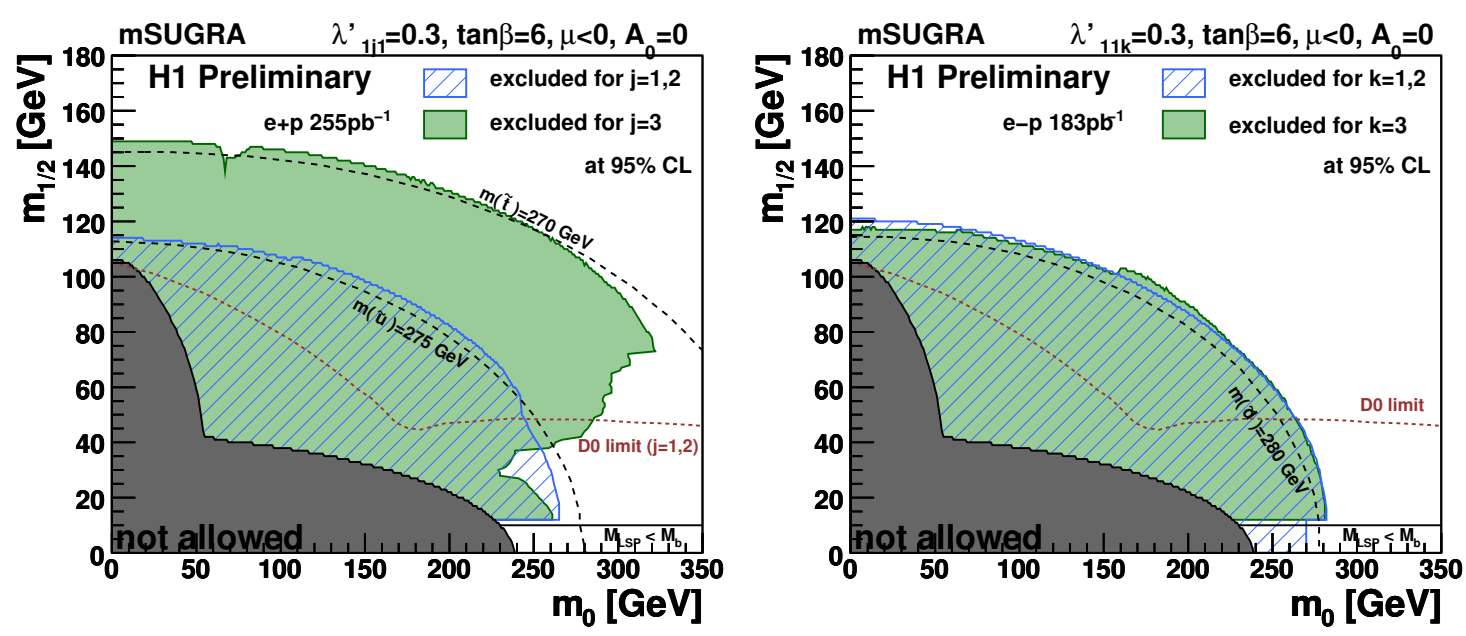

Figure 2: Exclusion limits at $95 \% \mathrm{CL}$ in the $m_{0}, m_{1 / 2}$ plane for $\tan \beta=6$ assuming (a) $\lambda_{1 j 1}^{\prime}=0.3$ and (b) $\lambda_{11 k}^{\prime}=0.3$ for $j, k=1,2$ hatched blue and $j, k=3$ filled green. Curves of constant squark mass are illustrated for $m(\tilde{u})=275 \mathrm{GeV}, m(\tilde{t})=270 \mathrm{GeV}$ and $m(\tilde{t})=290 \mathrm{GeV}$. Constraints obtained by the L3 experiment at LEP and D0 experiment at the Tevatron are also indicated.

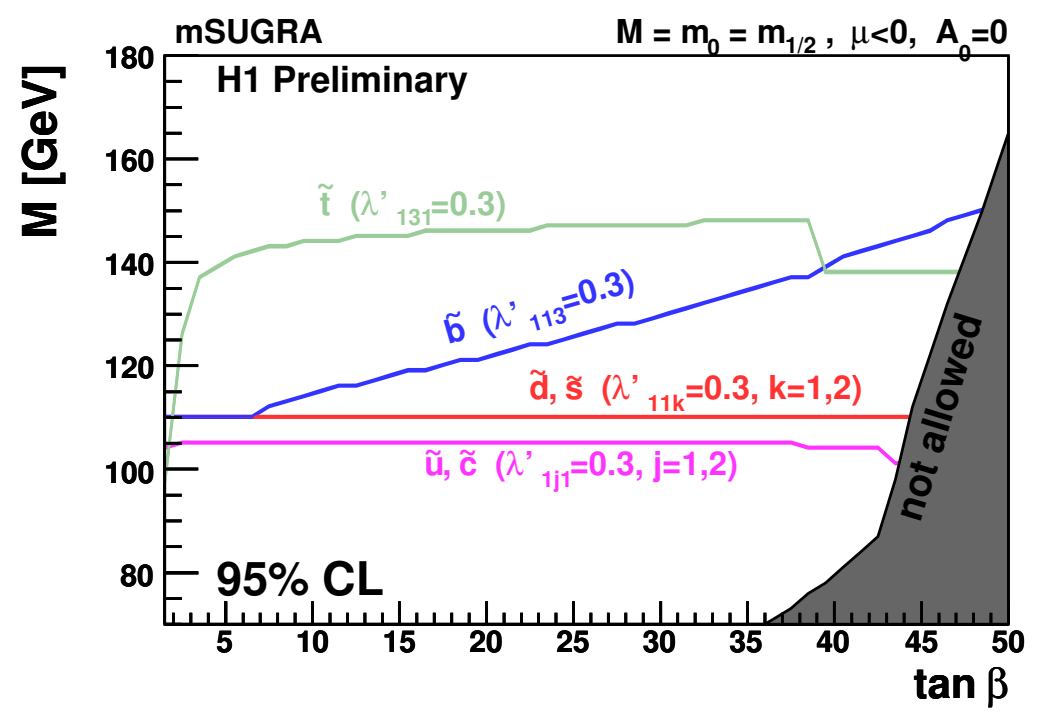

Figure 3: Exclusion limits for $m_{0}=m_{1 / 2}=M$ in the mSUGRA model as a function of $\tan \beta$. Shown are the $95 \% \mathrm{CL}$ exclusion limits for $\lambda_{1 j k}^{\prime}=0.3$. The area below the curves is excluded. The dark region corresponds to values of parameters where REWSB is not possible or the LSP is a sfermion. 